\title{
Identifying Patient Candidates for IL-1 Inhibition: Lessons From Real-World Cases
}

\author{
Annalina Avrama , Cátia Duarte ${ }^{b}$, Maria José Santosc, Charalampos Papagoras ${ }^{d}$, Konstantinos \\ Ritise, Roberto Scarpionif, Wolfgang A. Schmidtg, Panagiotis Skendros ${ }^{\mathrm{h}}$ \\ ${ }^{a}$ Medical Doctor; Immanuel Krankenhaus Berlin, Medical Centre for Rheumatology Berlin - Buch, Berlin, Germany \\ ${ }^{b}$ Rheumatologist, Rheumatology Department, Centro Hospitalar, Universitario de Coimbra, Coimbra, Portugal \\ 'Medical Doctor, Department of Rheumatology, Hospital Garcia de 'Orta, Lisbon, Portugal \\ University Hospital of Alexandroupolis, Alexandroupolis, Greece \\ ${ }^{d}$ Rheumatologist, First Department of Internal Medicine and Laboratory of Molecular Hematology, Democritus University of Thrace, \\ University Hospital of Alexandroupolis, Alexandroupolis, Greece \\ eProfessor of Internal Medicine, First Department of Internal Medicine and Laboratory of Molecular Hematology, Democritus University of Thrace, \\ University Hospital of Alexandroupolis, Alexandroupolis, Greece \\ ${ }^{f}$ Head, Department of Nephrology and Dialysis, Ospedale AUSL “Guglielmo da Saliceto", Piacenza, Italy \\ ${ }^{g}$ Deputy Director, Immanuel Krankenhause Berline Medical Centre for Rheumatology Berlin - Buch, Berlin, Germany \\ ${ }^{\text {h} A s s i s t a n t ~ P r o f e s s o r ~ o f ~ I n t e r n a l ~ M e d i c i n e, ~ F i r s t ~ D e p a r t m e n t ~ o f ~ I n t e r n a l ~ M e d i c i n e ~ a n d ~ L a b o r a t o r y ~ o f ~ M o l e c u l a r ~ H e m a t o l o g y, ~ D e m o c r i t u s ~ U n i v e r s i t y ~ o f ~ T h r a c e, ~}$ \\ University Hospital of Alexandroupolis, Alexandroupolis, Greece
}

\section{Keywords:}

Anakinra

Anti-IL-1 $\beta$ therapy

Canakinumab

Gouty arthritis

Tophaceous gout

\section{A B STRACT}

A subgroup of patients with gouty arthritis have a chronic recurring form that is particularly difficult to treat. Such patients experience repeated flares and often have abundant tophi. Many also have underlying comorbidities, such as renal impairment, cardiovascular disease, gastrointestinal disorders, obesity, and hypertension, which contraindicate the use of standard anti-inflammatory medications. Five patients with difficult to treat gouty arthritis who were either candidates and/or treated with anti-IL therapy are described.

C 2015 Société française de rhumatologie. Published by Elsevier Masson SAS. All rights reserved.

\section{IL-1 Inhibition in Systemic Inflammatory Syndrome Due to Gouty Arthritis}

\subsection{Introduction}

In addition to the rising prevalence of polyarticular involvement in gouty arthritis, an increasing number of patients with severe systemic inflammatory syndrome due to gouty arthritis are being admitted to hospitals. However, few reports have been published on this condition [1]. Here we present one of several such cases admitted to our hospital department for acute rheumatology and clinical immunology.

\subsection{Case Report}

A 68-year-old man was admitted at night on October 2, 2013 from an emergency unit of another hospital to the Medical Centre for Rheumatology Berlin-Buch with undefined inflammation and polyarthritis. He had had low-grade fever with chills and night sweats for 2 weeks, and his overall health condition was severely impaired. He had lost $5 \mathrm{~kg}$ within the past 8 weeks and was unable to rise and walk. Five days before admission, he had received clindamycin due to suspected erysipelas of the lower legs, resulting in an allergic rash. Deep vein thrombosis was excluded by ultrasound.

*Corresponding author.

E-mail address: w.schmidt@immanuel.de (W.A. Schmidt).

(c) 2015 Société française de rhumatologie. Published by Elsevier Masson SAS. All rights reserved.

Article produced with the institutional support of Novartis. 
Since 1985 , the patient has had a history of gout attacks with typical podagra and had been taking a daily dose of $100 \mathrm{mg}$ allopurinol. He also took colchicine on demand when experiencing a gout attack. Over the past year, he developed multiple visible tophi. Previous uric acid levels were not available. He consumed approximately 1 liter of beer per day. In addition, the patient was treated with amlodipine and ramipril for arterial hypertension, and with gabapentin for epilepsy. Renal colic has not been noted.

On physical examination, the patient appeared severely ill with multiple joint swellings and tophi, particularly of both first metatarsophalangeal (MTP) joints, ankle joints, knee joints, third right proximal interphalangeal (PIP) joint, first and second left metacarpophalangeal (MCP) joints, and elbows (Fig. 1). Both first MTP joints, the third right PIP joint, the first left MCP joint, and both knees were particularly reddened and hot. The patient was $176 \mathrm{~cm}$ tall and weighed $73 \mathrm{~kg}$.

Laboratory findings at admission were as follows: C-reactive protein $(\mathrm{CRP})$ level $=267 \mathrm{mg} / \mathrm{L}$; erythrocyte sedimentation rate $=$ $125 \mathrm{~mm} / \mathrm{h}$; uric acid level $=8.1 \mathrm{mg} / \mathrm{dL}(480 \mu \mathrm{mol} / \mathrm{L})$; and serum creatinine $=$ normal, with an estimated glomerular filtration rate $=78 \mathrm{~mL} / \mathrm{min}$.
Radiography showed typical erosions and tophi at the both first MTP joints as well as at the first left MCP joint. Ultrasound of the knees and the first toes showed typical features of gout, ie, tophi, aggregates, double contour sign, and erosions (Fig. 2) [2,3]. Double contour was detected in both first MTP joints and at the inter-condylar femoral region of both knees. Tophi were noted in both first MTP joints and at both quadriceps and patellar tendons. Ultrasound also revealed bilateral kidney stones. Ultrasound guided aspiration of the left first MTP joint revealed $2 \mathrm{~mL}$ of dense white fluid. Multiple urate crystals could be seen by polarizing light microscopy in the aspirate. Gram stain and bacterial culture were negative.

The patient received daily colchicine $1.5 \mathrm{mg}$ throughout hospitalization. Because his symptoms did not improve, daily oral prednisolone $50 \mathrm{mg}$ was added, followed by daily intravenous prednisolone $70 \mathrm{mg}$. His symptoms and CRP values (Fig. 3) did not significantly improve. Blood and aspirate cultures were negative. Subsequently (from October 16-22), the patient received daily subcutaneous (sc) anakinra $100 \mathrm{mg}$. His systemic symptoms, pain, and joint redness improved the day after the first injection. His CRP level dropped to $28 \mathrm{mg} / \mathrm{L}$ on October 20. Treatment with febuxostat $120 \mathrm{mg} /$ day was initiated on October 22 . The patient
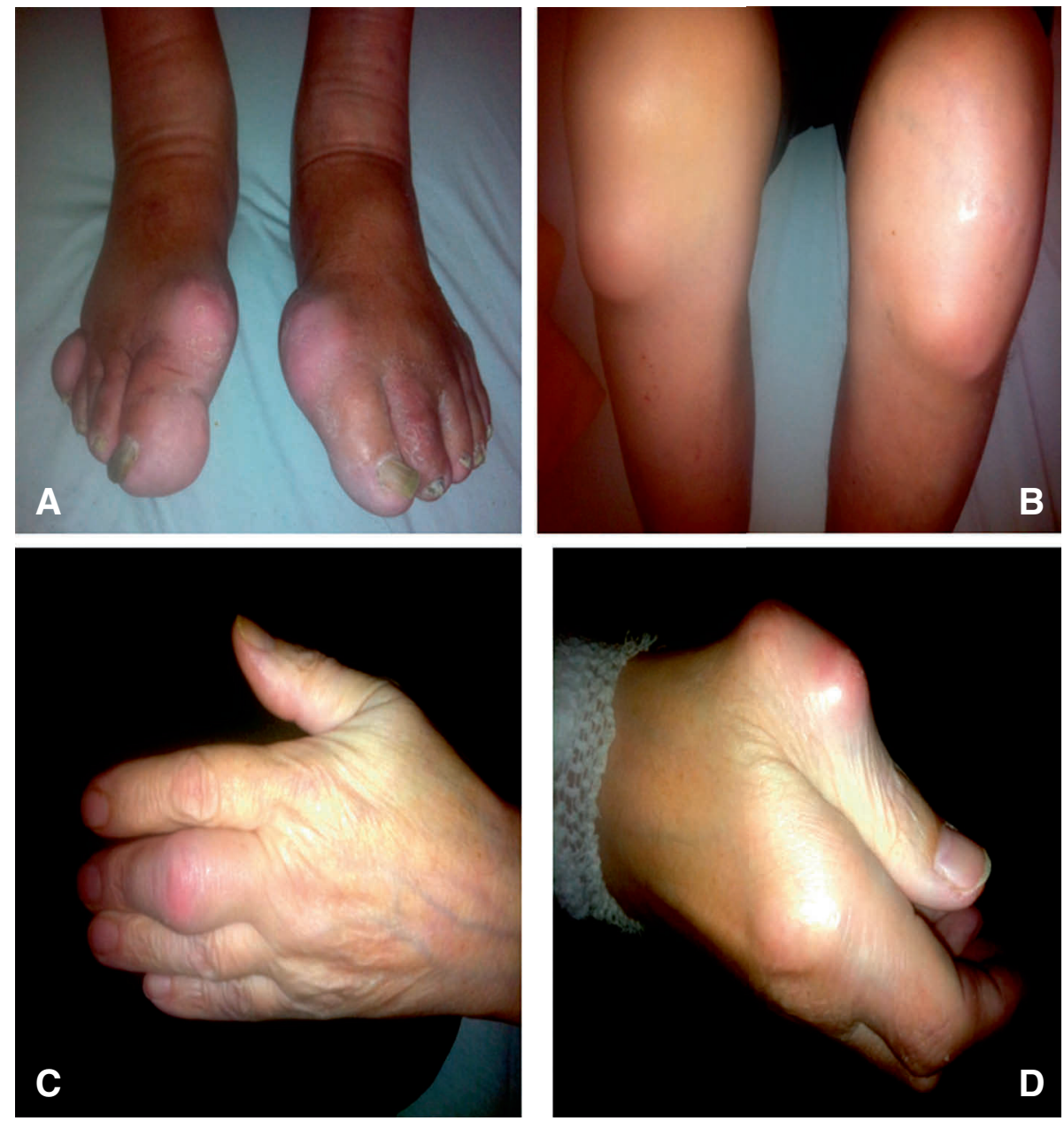

Figure 1. Acute polyarticular tophaceous gout with A) red swollen MTP 1 joints; B) bilateral prepatellar tophi; C) inflamed right PIP 3 joint; and D) inflamed left MCP joint with deformity.

MCP, metacarpophalangeal; MTP, metatarsophalangeal; PIP, proximal interphalangeal. 
was informed about dietary issues and stopped drinking beer. On October 23, following final negative results for bacterial cultures and tuberculin tests, he received sc canakinumab $150 \mathrm{mg}$.

The patient remained well and had no adverse effects from the newly initiated medications. Prednisolone was tapered. No colchicine was given. On November 26, his CRP level was $12 \mathrm{mg} / \mathrm{L}$ and uric acid level was $5.5 \mathrm{mg} / \mathrm{dL}$ (330 $\mu \mathrm{mol} / \mathrm{L})$. On December 7, he had a gout attack of a first MTP joint for which he took colchicine $1.5 \mathrm{mg}$. His symptoms resolved the next day and he remained on daily colchicine $1 \mathrm{mg}$.

On January 16,2014 , the patient visited the office to ascertain as to whether another canakinumab injection would be indicated following the 12 weeks that passed since the first injection was administered. Since colchicine had been reinitiated, he had no additional gout attacks. Painless tophi and joint swellings without redness were still present. His CRP level was $2 \mathrm{mg} / \mathrm{L}$ and uric acid level was $5.5 \mathrm{mg} / \mathrm{dL}(326 \mu \mathrm{mol} / \mathrm{L})$. Treatment with colchicine $1 \mathrm{mg} /$ day and febuxostat $120 \mathrm{mg} /$ day was continued, and the patient did not receive additional canakinumab injections.

In April 2014, the patient had 1 minor gout attack of a first MTP joint. On April 28, his CRP level was $8 \mathrm{mg} / \mathrm{L}$ and uric

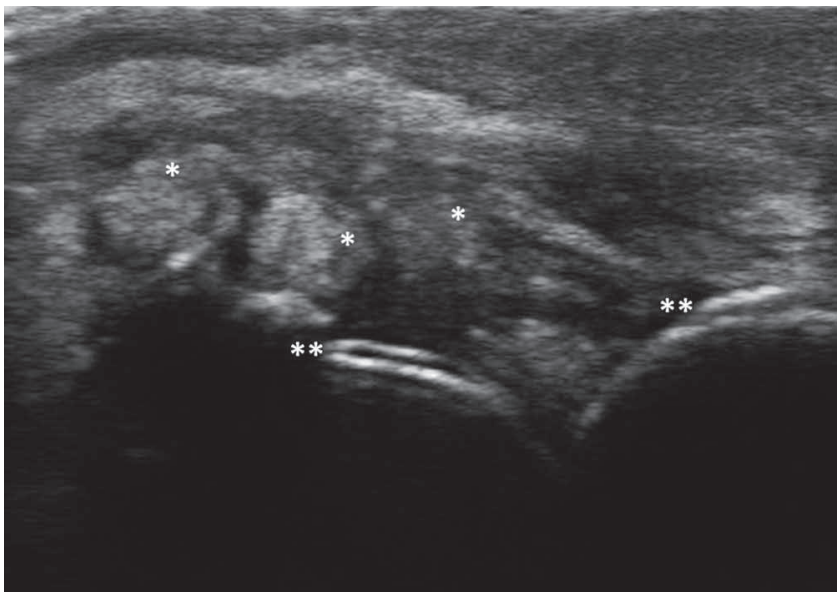

Figure 2. Ultrasound image of the right MTP 1 joint showing multiple tophi $\left({ }^{*}\right)$ and double contour sign $\left({ }^{* *}\right)$.

MTP, metatarsophalangeal. acid level was $4.5 \mathrm{mg} / \mathrm{dL}(272 \mu \mathrm{mol} / \mathrm{L})$. Treatment remained unchanged. No further gout attacks occurred until July 24 when his CRP level was $2 \mathrm{mg} / \mathrm{L}$ and his uric acid level was $3.5 \mathrm{mg} / \mathrm{dL}$ $(213 \mu \mathrm{mol} / \mathrm{L})$. The febuxostat dose was subsequently reduced to $80 \mathrm{mg} /$ day. The patient regained the weight he had lost during the acute phase of the disease and remained at a weight of $78 \mathrm{~kg}$ throughout the time of observation.

Until January 2015, the patient was taking prophylaxis colchicine $0.5 \mathrm{mg} /$ day (reduced from $1 \mathrm{mg} /$ day since October 2014) and reported no further gout attacks. Joint swelling and tophi significantly decreased. His CRP levels remained normal and his uric acid levels remained $<5.0 \mathrm{mg} / \mathrm{dL}(300 \mu \mathrm{mol} / \mathrm{L})$.

\subsection{Discussion}

Systemic inflammatory syndrome due to gouty arthritis appears to be under reported in the literature. These patients are often unsuccessfully treated for suspected infection. Many of them improve with colchicine or glucocorticoid therapy. However, some patients, as the one we report on, are resistant to such treatment. Interleukin-1 (IL-1) inhibition is an option for these patients.

Rilonacept, canakinumab, and anakinra have been used successfully in the treatment of patients with gouty arthritis. In randomized, controlled trials, both rilonacept and canakinumab have been shown to prevent flares in patients initiating uratelowering therapy (ULT) [4,5]. However, only canakinumab has consistently shown in phase III trials to be effective treatment for acute flares and to reduce the risk of new flares in patients with difficult-to-treat disease [6]. Anakinra has shown benefit in the treatment of patients with acute and chronic gouty arthritis in pilot studies, retrospective reviews, and case reports [7].

Canakinumab is the only anti-IL-1 inhibitor approved for gouty arthritis. In 2013, canakinumab was approved in the European Union for the treatment of patients with frequent gouty arthritis attacks ( $\geq 3$ attacks in the previous 12 months) in whom NSAIDs and colchicine are contraindicated, are not tolerated, or do not provide an adequate response, and in whom repeated courses of corticosteroids are not appropriate [8].

After failure of NSAIDs, colchicine, and prednisolone treatment, we started our patient on anti-IL therapy with anakinra

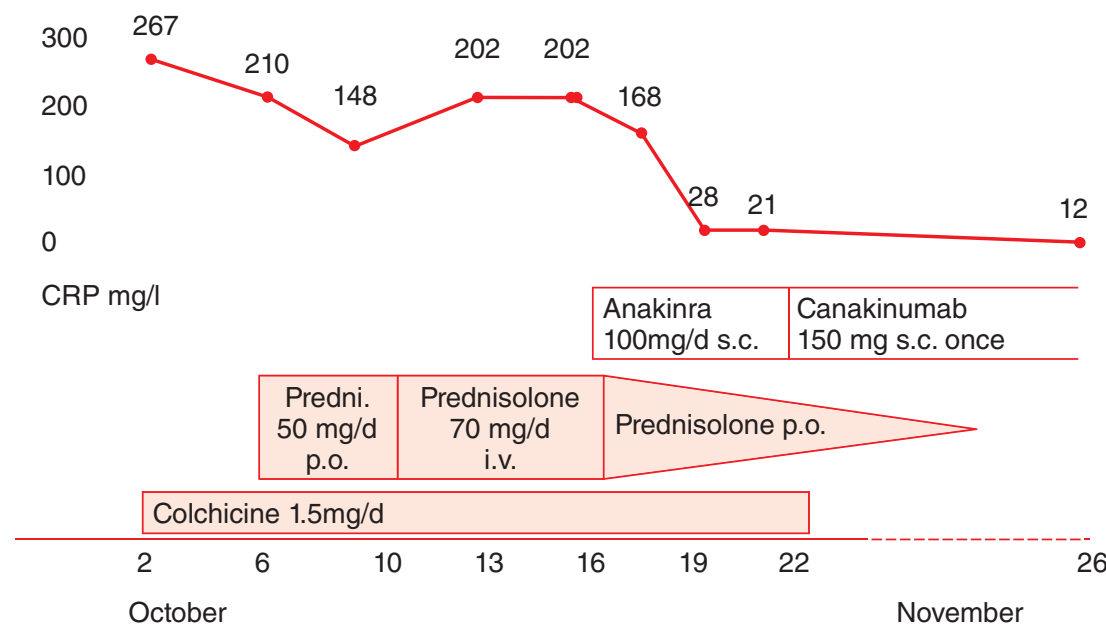

Figure 3. Medication and CRP levels at the acute phase of the disease.

CRP, C-reactive protein. 
(off-label use), which has a relatively short half-life, to determine if IL-1 inhibition would be effective for our patient and to rule out acute bacterial infection and chronic tuberculosis. We then administered 1 injection of canakinumab in an outpatient setting to continue the anti-inflammatory effect. Although febuxostat treatment was initially started with the higher approved dose of $120 \mathrm{mg} /$ day to reduce the urate load as soon as possible, paradoxical flares did not occur in the first weeks of ULT.

In patients who respond to canakinumab and require retreatment, there should be an interval of at least 12 weeks before a new dose of canakinumab can be administered on demand during an attack [8]. In our patient, colchicine prophylaxis was sufficient for preventing major gout attacks and systemic inflammatory syndrome over the course of time. Of note, canakinumab is not approved for flare prophylaxis, rather only for treatment of acute flares.

In conclusion, inflammatory syndrome due to gouty arthritis indeed exists, and additional case reports or case series need to be published. IL-1 inhibition appears to be effective for the condition.

\section{Anti-IL-1 Therapy Suppresses Inflammation and Provides an Opportunity to Optimize ULT in a Patient with Difficult-to-Treat Gouty Arthritis}

\subsection{Introduction}

Gouty arthritis, the most common inflammatory arthritis worldwide, is caused by chronic hyperuricemia and tissue deposition of monosodium urate crystals (MSU). The condition occurs predominantly in men and older women. Its prevalence has been increasing in developed counties, with approximately 1 in 40 adults in the United Kingdom being affected [9]. Acute arthritis is the most common feature leading to diagnosis. If the hyperuricemia is not adequately treated at the stage of intermittent acute flares, gout can progress to a chronic and destructive polyarthritis, which can mimic rheumatoid arthritis (RA).

The human and economic burden of gouty arthritis is high and continues to rise with the aging of the population, increasing rates of obesity, and lifestyle changes [10]. Many patients with gouty arthritis have comorbidities, either at diagnosis or during the course of the disease [11]. Cardiovascular, renal, and metabolic disorders most commonly coexist in patients with gouty arthritis. Additionally, malignancies, gastrointestinal disorders, and other diseases are overrepresented in these patients [11].

The management of patients with gouty arthritis is often suboptimal, particularly in patients with multiple comorbidities, who are particularly difficult-to-treat $[9,12]$. We present a patient with chronic tophaceous gout who has multiple comorbidities, limiting the use of standard therapies.

\subsection{Case Report}

A 48-year-old Caucasian man was referred to the outpatient rheumatology clinic with suspected RA. He reported that since the age of 35, he has had painful joint symptoms, which initially were localized to the lower limbs but more recently involved the hands, wrists, and elbows. Initially, his symptoms were episodic, subsided spontaneously, and did not significantly interfere with his daily activities. Within the following years, the painful episodes gradually became longer in duration and currently, the pain is almost constant. He noticed the appearance of slightly painful subcutaneous (sc) nodules on his fingers and elbows, progressive deformation of his fingers, and limitation of his wrist, ankle, and knee movements that interfered with his daily activities.

The patient previously worked as a policeman, performing mostly administrative duties. He had been consuming 0.5 liter of wine and 1 to 2 beers daily. He has a history of hypertension that was controlled with lisinopril and hydrochlorothiazide 20/12.5 mg/day. For several years, he used over-the-counter nonsteroidal anti-inflammatory drugs (NSAIDs) to control joint pain. Because NSAIDs were no longer providing sufficient pain relief, he recently sought medical care. After consulting his general practitioner, he was diagnosed with symmetric deforming polyarthritis and was started on prednisolone $10 \mathrm{mg} /$ day. His family history was unremarkable.

On physical examination, the patient had a mild limping gait. His blood pressure was 163/98 mm Hg, heart rate was 72 bpm, and body mass index was $31 \mathrm{~kg} / \mathrm{m}^{2}$. Multiple firm nontender sc nodules were present on both elbows and dorsum of the fingers. Ulcerated nodules on the pulps of his left thumb and third to fifth fingers were also present (Fig. 4). The range of motion of his shoulders, elbows, knees, ankles, and subtalar joints was limited, and his knees, wrists, metacarpophalangeal (MCP) joints and some proximal interphalangeal (PIP) joints were tender and swollen. The remainder of his physical examination was normal

$\mathrm{X}$-ray films of the patient's hands and feet showed multiple joint deformities and extensive bony erosions of the wrist, and MCP, PIP, (Fig. 5), and metatarsophalangeal joints. The complete blood count was normal, as were blood levels of alanine aminotransferase, albumin, globulin, and alkaline phosphatase. Other test results are shown in Table 1 . The estimated glomerular filtration rate was $49 \mathrm{~mL} / \mathrm{min}$.

A diagnostic arthrocentesis of his knee was performed and analysis of the synovial fluid showed a cell count of 37,000 cells/ $\mathrm{mm}^{3}$, predominantly polymorphonuclears; Gram staining was negative. Numerous MSU crystals were identified under polarized light microscopy. Aspiration of $1 \mathrm{sc}$ nodule also revealed the presence of MSU crystals. The diagnosis of chronic tophaceous gout was established, and treatment goals included symptomatic control of polyarthritis, reduction of uricemia, and prevention of further attacks.

Considering the patient's comorbidities (metabolic, cardiovascular, and renal disorders), NSAIDs were discontinued and low-dose colchicine was prescribed $(0.5 \mathrm{mg} / \mathrm{day})$. To control his hypertension, losartan $100 \mathrm{mg} /$ day was substituted for lisinopril/hydrochlorothiazide. Urate-lowering therapy (ULT) with allopurinol $50 \mathrm{mg}$ /day was started, and tapering/discontinuation of glucocorticoids was planned as soon as satisfactory pain relief could be achieved. Lifestyle changes were strongly encouraged, namely stopping regular alcohol intake and reducing body weight.

The patient continued to have persistent pain in his knees and ankles that severely limited walking. In addition, he continued to experience frequent flares that prevented optimal ULT dosing. After 4 months of treatment, elevated uricemia persisted, 

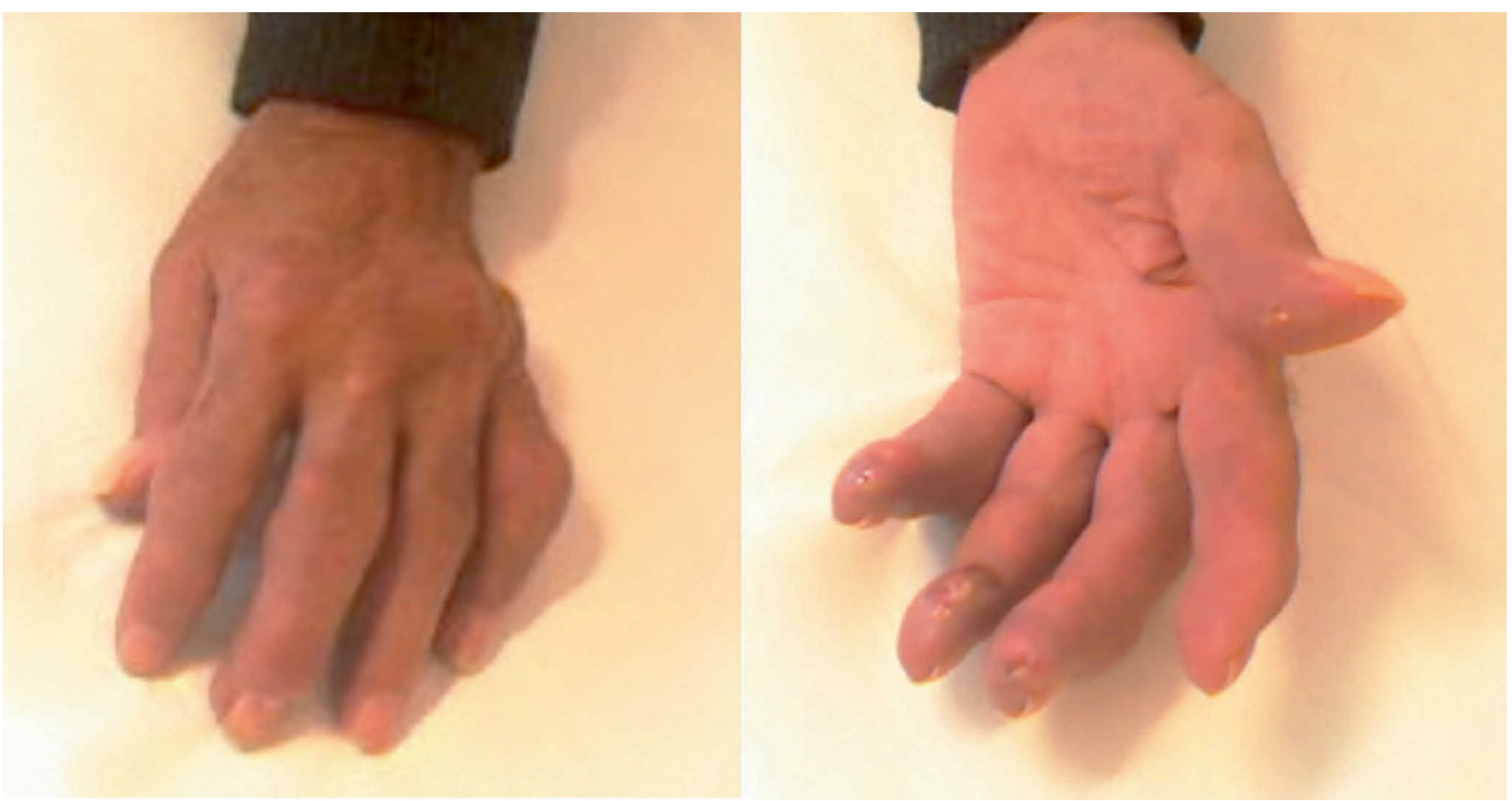

Figure 4. Patient's left hand with finger tophi and ulcerations of digital pulps.
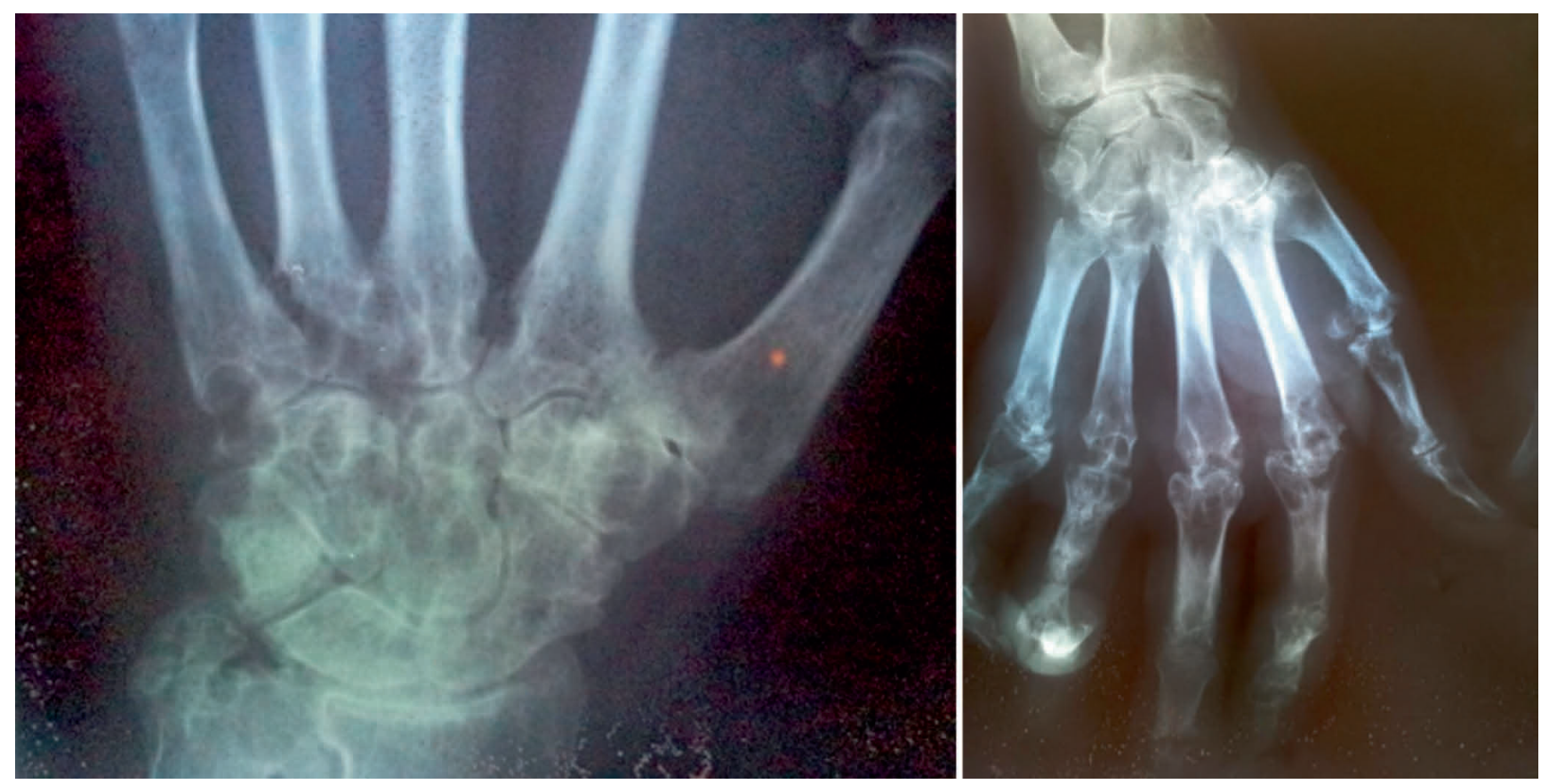

Figure 5. Hand radiographs show destructive arthropathies of the wrist and MCP joints $\mathrm{MCP}$, metacarpophalangeal.

polyarticular gouty attacks continued, and treatment adherence was poor. At this point, interleukin (IL)-1 inhibition was considered, and the patient consented to a trial of anakinra $100 \mathrm{mg} /$ day sc (the only IL- 1 antagonist commercially available at that time in Portugal [approved for the indication of moderate to severe rheumatoid arthritis]). Anakinra led to a rapid and complete resolution of symptoms, permitting the tapering and eventual discontinuation of steroids, and a gradual dose increase of ULT.
Anakinra was initially administered for 1 week and on demand thereafter (for 2-3 days to abrogate gouty flares). One year later, the patient was clear of arthritis, his serum urate level was controlled, and his renal function remained stable. He continues to have moderate pain in his ankle and foot after exercising that can be managed without the use of analgesics. He also continues to have limitation of movement of his shoulders, elbows, fingers, and subtalar joints but is able to continue to work. 
Table 1

Laboratory Results

\begin{tabular}{lll}
\hline ESR & $36 \mathrm{~mm} / \mathrm{h}$ & $\mathrm{N}<16 \mathrm{~mm} / \mathrm{h}$ \\
Fasting blood glucose & $158 \mathrm{mg} / \mathrm{dL}$ & $\mathrm{N}<110 \mathrm{mg} / \mathrm{dL}$ \\
Serum creatinine & $2.4 \mathrm{mg} / \mathrm{dL}$ & $\mathrm{N}<0.9 \mathrm{mg} / \mathrm{dL}$ \\
Serum uric acid & $11.7 \mathrm{mg} / \mathrm{dL}$ & $\mathrm{N}<6.8 \mathrm{mg} / \mathrm{dL}$ \\
IgM RF & Negative & \\
ACPA & Negative & \\
\hline
\end{tabular}

ACPA, anti-citrullinated protein antibodies; ESR, erythrocyte sedimentation rate; IgM, immunoglobulin M; N, normal; RF, rheumatoid factor.

\subsection{Discussion}

This case illustrates the potential management challenges in patients with gouty arthritis who have multiple comorbidities. The therapeutic goals for gouty arthritis are control of acute arthritis, prevention of future attacks, and control of comorbidities. In the present case, a long period of time had elapsed between the patient's initial symptoms and diagnosis, and for many years, the patient had used NASIDs regularly without appropriate monitoring.

Gastrointestinal, cardiovascular, and renal side effects are associated with chronic NSAID use [13], and although NSAIDs constitute the first line of treatment for acute gouty arthritis, their use is not recommended when renal function is impaired [14]. Likewise, the use of corticosteroids is not the most appropriate treatment given the risk of worsening the metabolic control and aggravating cardiovascular risk. Colchicine may be an alternative for patients who cannot tolerate NSAIDs, but the dosage should be reduced in patients with renal failure. Colchicine can be useful for controlling acute attacks as well as for preventing flares when initiating ULT. Unfortunately, this was not the case in our patient, who continued to experience gouty flares despite taking colchicine and prednisolone, especially when trying to increase the allopurinol dose.

Patients with gouty arthritis and multiple coexisting comorbidities have limited treatment options and can be particularly challenging to treat. Recently, IL-1 inhibition has been shown to be effective for controlling acute arthritis in patients intolerant or refractory to conventional therapies $[6,15]$. Canakinumab has been approved in the European Union for patients with frequent gouty arthritis attacks who cannot be managed with standardof-care medication. However, it was not available in Portugal at the time our patient was being treated. Anakinra, which proved effective, was the only available IL-1 inhibitor.

A few case reports in the literature document the efficacy of ankinra in acute gout but its use remains off-label [15]. In the present case, IL-1 inhibition was crucial to abrogate inflammation and allow the successful introduction of effective doses of allopurinol and the discontinuation of steroids. Additionally, this therapeutic approach contributed to the patient's improved self-confidence and adherence to treatment. The most challenging aspect of the treatment plan is the long-term adherence to urate lowering drugs and implementation of lifestyle changes, including healthy eating, eliminating alcohol beverages, and weight control, which are crucial not only for gout control but especially for reducing cardiovascular disease risk.

In summary, gouty arthritis management should address the patient as a whole and take into account the presence of comorbidities. IL-1 inhibition represents a significant advance in the treatment of patients with limited therapeutic options.

\section{Treatment Dilemma in Gouty Arthritis: Managing a Patient With Complex Comorbidities and Intolerance to Standard Therapies}

\subsection{Introduction}

Gouty arthritis is an ancient disease. Despite significant advances in the understanding of its risk factors, etiology, pathogenesis, prevention, and treatment, millions of people diagnosed with gout have repeated attacks of acute arthritis and other complications. Comorbid conditions, that often accompany gout, present challenges when managing the condition [16].

\subsection{Case Report}

A 70-year-old man with tophaceous gout and a 20-year history of gouty arthritis was referred to our rheumatology outpatient clinic. He reported a history of recurrent acute monoarthritis involving his first metatarsophalangeal joint. Eventually, he developed more severe joint involvement, with polyarthritis, shorter inter-critical periods, and tophus development (Fig. 6). He now experiences frequent polyarthritis attacks (2-3 per month).

Over the years, the patient developed multiple comorbidities including type 2 diabetes mellitus, hypertension, dyslipidaemia, chronic kidney disease (glomerular filtration rate, $50 \mathrm{~mL} / \mathrm{min}$ ), and upper gastrointestinal (GI) bleeding and a peptic ulcer associated with NSAID overuse. Five years ago, he was diagnosed with monoclonal gammapathy of undetermined significance (MGUS), and in 2012, he had an acute myocardial infarction.

Initially, the patient achieved a good clinical response with the use of nonsteroidal anti-inflammatory drugs (NSAIDs) or colchicine. However, due to the development peptic ulcer disease and a high risk of cardiovascular disease (history of an acute event), NSAIDs were subsequently contraindicated. He also developed GI intolerance (diarrhoea) to colchicine when the dose used was greater than $1 \mathrm{mg} /$ day. Low-dose colchicine was not effective in controlling his acute episodes. To control the frequent severe attacks, the patient was prescribed glucocorticoid (GC) therapy (30 mg/day) with dose tapering (30, 20, 10, 5 mg/day) every 3 days until discontinuation. GC therapy resulted in uncontrolled hyperglycaemia, but this was the only available treatment option for our patient.

Allopurinol (100 mg/day), in conjunction with prophylactic colchicine $(0,5 \mathrm{mg} /$ day), was also initiated. However, the patient developed a severe hypersensitivity with intense pruritus and skin lesions. Despite undergoing a desensitization program with antihistamine treatment, he was unable to tolerate a dose greater than $12.5 \mathrm{mg} /$ day of allopurinol. No other urate-lowering therapy (ULT) is available in Portugal.

Despite making lifestyle changes (low-purine diet, no alcohol consumption, good hydration, and weight loss), taking vitamin C 

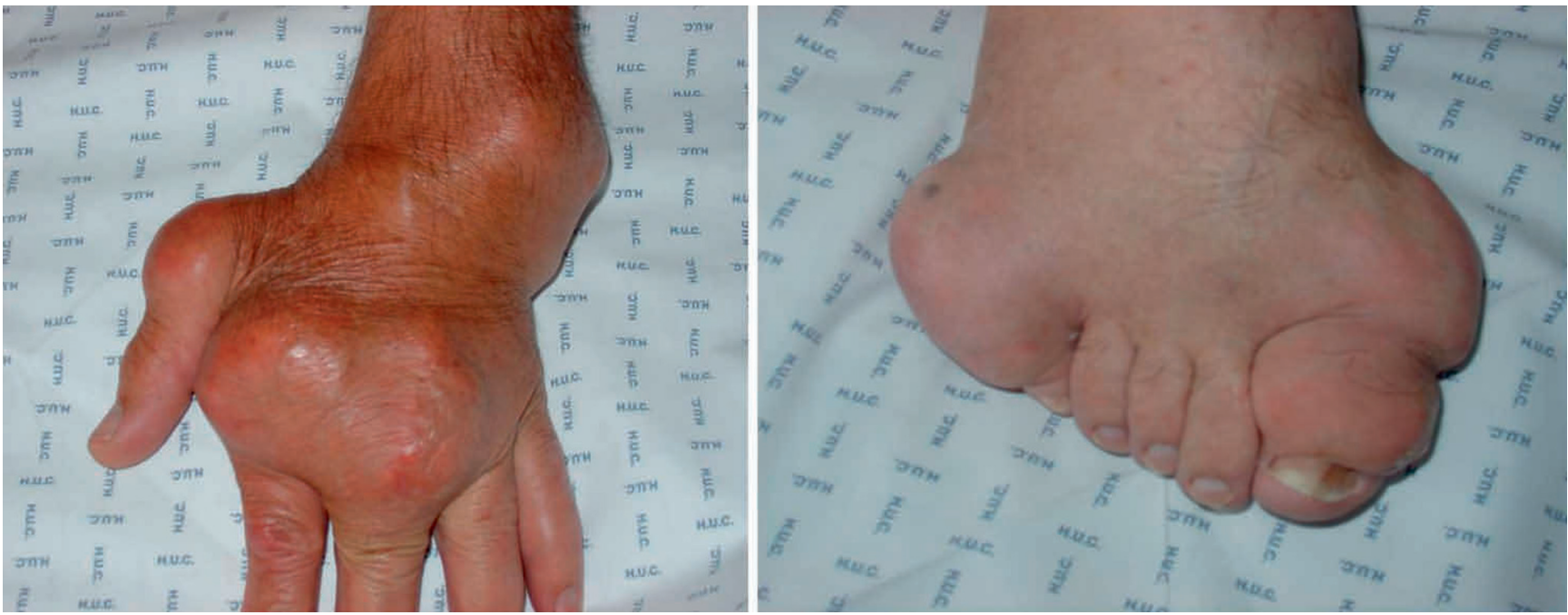

Figure 6. Tophi in the patient's hand and foot

supplements, receiving medications associated with uricosuric properties (losartan $50 \mathrm{mg} /$ day and fenofibrate $267 \mathrm{mg} /$ day) to control comorbidities, and avoiding hyperuricemic drugs (diuretic agents and low-dose aspirin), the patient's acid uric levels remain persistently elevated $(12.1 \mathrm{mg} / \mathrm{dl})$.

\subsection{Discussion}

Herein we report on a patient with complicated gouty arthritis who remains untreated due to limited treatment options because of his multiple comorbidities. Choosing appropriate treatment strategies for these types of patients deserves further discussion.

Based on treatment guidelines/recommendations for gouty arthritis [14, 17-19], colchicine and NSAIDs are first-line treatment options for acute gout attacks. In 2 randomized clinical trials $[20,21]$, colchicine was shown to be superior compared with placebo in the treatment of acute gout. In one of those trials, early self-administered low-dose ( $1.8 \mathrm{mg} / 1$ hour) colchicine yielded efficacy in gout flare management comparable with that of high-dose ( $4.8 \mathrm{mg} / 6$ hours) colchicine, with a safety profile indistinguishable from that of placebo [21]. Several trials comparing various NSAID agents in the management of gouty arthritis found no significant differences in efficacy among the agents [17]. The choice between colchicine and NSAIDs is driven by patient's preference and/or contraindications to a specific agent. In patients with severe gouty arthritis, the American College of Rheumatology (ACR) guidelines recommend NSAIDs in combination with colchicine [19]. In our patient, traditional NSAIDs were contraindicated due to a history of GI bleeding. COX-2 inhibitors were avoided due to the patient's high cardiovascular risk.

For patients with an acute severe gout attack, the ACR guidelines recommend corticosteroids alone or in combination with colchicine [19]. The route of administration depends on the number and type of joints involved. Oral corticosteroids are recommended for polyarticular involvement, and intra-articular corticosteroids are recommended when 1 to 2 large joints are involved [19]. Due to the severe polyarticular involvement in our patient, we prescribed a short course of a medium-to-high dose of prednisone (30 mg/day, with tapering) that provided good control of his symptoms but negatively impacted control of his diabetes.

New alternative treatments are now available for patients with severe gouty arthritis refractory to other agents. Biologic IL-1 inhibitors agents, such as canakinumab (approved in the European Union), can be considered. In a phase III, randomized, controlled trial (RCT), canakinumab $150 \mathrm{mg}$ provided significantly greater and more rapid reduction in pain and signs and symptoms of inflammation compared with triamcinolone acetonide $40 \mathrm{mg}$. Improvement in health-related quality of life occurred in both treatment groups but with a faster onset with canakinumab $150 \mathrm{mg}$ compared with triamcinolone acetonide $40 \mathrm{mg}$ [22], confirming the results of a phase II dose-finding, RCT, in which canakinumab $150 \mathrm{mg}$ provided rapid and sustained pain relief in patients with acute gouty arthritis, and significantly reduced the risk of recurrent flares compared with triamcinolone acetonide [23].

Given our patient's severe acute gout attacks and his limited treatment options due to comorbidities, canakinumab may be a promising alternative to standard therapy. Because patients with MGUS have a risk of progression to multiple myeloma or related malignancy, we must carefully weigh the benefits versus the risks of canakinumab use. At this time, no safety data exists for the use of canakinumab in patients with MGUS and other plasma cell neoplasms.

Another important component in the management of patients with gouty arthritis is the use of chronic ULT. Xanthine inhibitors, such as allopurinol or febuxostat, are considered first-line ULT $[14,17,18,24]$. Taking into consideration the efficacy, safety, and costs of allopurinol, there is a strong consensus in using it as the first option [17]. Low-to-medium doses (80 or $120 \mathrm{mg} /$ day) of febuxostat can be considered in patients who do not respond or are contraindicated to allopurinol. Uricosuric agents, such as probenecid or benzobromarone, can be considered when xanthine-oxidase inhibitors do not achieve adequate control. Pegloticase is efficacious but is associated with an increased risk of acute attacks and infusion reactions, and its use should be considered carefully $[17,25]$. Our patient had a severe hypersensitivity 
reaction to allopurinol. Febuxostat and uricosuric agents are not available in our country (Portugal). Upon special request to our regulatory authority, we were granted access to febuxostat. The patient received febuxostat $80 \mathrm{mg}$ day; his last uric acid level decreased to $6.2 \mathrm{mg} / \mathrm{dL}$ and he has had no new acute attacks.

Although gouty arthritis is a common disease and efficacious treatment is available for most patients, this case illustrates the challenges in managing the condition in patients with complex comorbidities and limited treatment options.

\section{Managing a Patient With Persistent Gout Flares Complicated by Comorbidities and Polypharmacy: An Evidence-based Approach}

\subsection{Introduction}

Gouty arthritis is a common type of arthritis that affects approximately $2 \%$ of the adult population. Interleukin- $1 \beta$ (IL$1 \beta$ )-mediated inflammation that is triggered by monosodium urate (MSU) crystals is the hallmark of the pathogenesis of a gout attack [26,27]. Recently, both the European League Against Rheumatism (EULAR) and the American College of Rheumatology (ACR) published guidelines and recommendations for the management of gout $[19,24,28]$. However, in daily practice, physicians often fail to effectively treat their patients, and patients fail to comply with their prescribed treatment - both contributing factors as to why some patients remain exposed to the long-term toxic consequences of accumulating urate crystals in joints, soft tissues, and elsewhere [29,30]. Moreover, patients with gouty arthritis often have multiple comorbidities, limiting the use of anti-inflammatory and urate-lowering drugs due to potential adverse drug reactions and interactions. Herein, we discuss the management of a real-life patient with recurrent gouty arthritis and multiple comorbidities.

\subsection{Case Report}

A 76-year-old Caucasian man presented to the outpatient office complaining of pain and swelling of his left wrist over the past few days. During the preceding year he had had several bouts of acute arthritis (left ankle, right ankle, right knee), all of which had been treated with paracetamol and topical application of ice. The resolution of each arthritis attack was slow and partial, and was shortly followed by a new attack.

The patient had multiple significant comorbidities. He was obese with a body mass index of $39.8 \mathrm{~kg} / \mathrm{m}^{2}$, and he had chronic obstructive pulmonary disease (COPD) due to past smoking, and sleep apnea syndrome. He also had arterial hypertension, type 2 diabetes mellitus, ischemic heart disease, chronic atrial fibrillation, and congestive heart failure. He was on fosinopril, amlodipine, hydrochlorothiazide, furosemide, digoxin, dabigatran, glibenclamide, sitagliptin, and montelukast.

On physical evaluation, the patient's blood pressure was $140 / 70 \mathrm{~mm} \mathrm{Hg}$, temperature was $36.7^{\circ} \mathrm{C}$, and weight was 254 pounds $(115 \mathrm{~kg})$. He had acute arthritis of the left wrist but no detectable tophi. Irregular heart tones and a significant bilateral lower limb edema were noted.

Laboratory tests revealed an unremarkable blood count and raised inflammatory markers (erythrocyte sedimentation rate,
$30 \mathrm{~mm} / \mathrm{h}$; C-reactive protein, $15 \mathrm{mg} / \mathrm{L}$ ). The serum biochemistry panel revealed elevated levels of glucose $(187 \mathrm{mg} / \mathrm{dL})$, urea (92 $\mathrm{mg} / \mathrm{dL})$, creatinine $(1.14 \mathrm{mg} / \mathrm{dL})$, urate $(11.5 \mathrm{mg} / \mathrm{dL})$, and mild hyponatremia (serum sodium, $132 \mathrm{mEq} / \mathrm{L}$ ); serum liver enzymes and creatine phosphokinase activities were within normal ranges. Serum lipid levels were also within target for the patient's profile (cholesterol, $155 \mathrm{mg} / \mathrm{dL}$; triglycerides, $87 \mathrm{mg} /$ $\mathrm{dL}$; high-density lipoprotein cholesterol, $28 \mathrm{mg} / \mathrm{dL}$ ).

Musculoskeletal ultrasound of the affected wrist showed at least grade 2 gray scale and power Doppler synovitis, both indicative of active arthritis. On joint aspiration, a small amount of synovial fluid was retrieved, showing an increased leukocyte count $\left(2400 / \mathrm{mm}^{3}\right)$, consisting mainly of neutrophils (58\%) and scattered needle-shaped crystals.

The patient was diagnosed with recurrent acute gouty arthritis and was prescribed oral methylprednisolone $(8 \mathrm{mg}$ daily for 5 days followed by tapering and discontinuation over the next 10 days) and colchicine $0.5 \mathrm{mg}$ tid. Long-term febuxostat $80 \mathrm{mg}$ daily was also prescribed, which was to begin after the attack subsided, along with maintenance colchicine for at least 3 months to prevent further attacks. Dietary measures were also recommended. A few days later, the patient reported an improvement of his arthritis. However, his blood glucose level had risen $>200 \mathrm{mg} / \mathrm{dL}$ and he was having bouts of diarrhea. The patient was advised to reduce the colchicine dosage to $0.5 \mathrm{mg}$ bid. Soon after, the gouty attack resolved fully; the patient started febuxostat while remaining on low-dose colchicine. However, 2 weeks later he experienced acute arthritis of the left ankle and received a new course of oral glucocorticoids while remaining on the same regimen of febuxostat and colchicine. Ten days after the resolution of the attack, another attack occurred at the third left metacarpophalangeal joint. Repeat serum biochemistry showed the patient's serum urate level had decreased to $8.51 \mathrm{mg} / \mathrm{dL}$ but was not yet optimal $(<6 \mathrm{mg} / \mathrm{dL})$. Therefore, it became apparent that the patient's therapeutic regimen needed to be modified. What are the most appropriate steps to take at this time?

\subsection{Discussion}

This case illustrates the potential management challenges for some patients with gouty arthritis referred to a tertiary care hospital, particularly by general practitioners or other specialists, as these patients have the most severe disease and/or major comorbidities. Evidence shows that at the time of diagnosis, patients with gouty arthritis have a significantly higher prevalence of comorbid conditions compared with matched non-gout controls, as well as a higher incidence of new comorbidities after the diagnosis of gout. In addition to the spectrum of metabolic syndrome comorbidities, these patients have a broad range of cardiovascular, genitourinary, endocrine, and other disorders. Furthermore, patients with gouty arthritis have a slightly increased mortality risk even after adjusting for baseline comorbidities and other confounding factors [11].

Treatment for gouty arthritis consists of addressing 3 aspects: 1) changing lifestyle factors that increase serum urate; 2) antiinflammatory treatment for the management and prevention of acute arthritis; and 3) urate-lowering therapy (ULT) [19,24,28].

The patient presented herein had significant cardiopulmonary disorders that make lifestyle changes challenging. COPD and 
congestive heart failure limit the ability for physical exercise and weight loss, whereas severe peripheral edema makes it difficult to reduce diuretics.

With regard to anti-inflammatory treatment, of the 3 first-line available options (nonsteroidal anti-inflammatory drugs [NSAIDs], colchicine, glucocorticoids), NSAIDs are the least attractive option for this patient, as they can impair the renal function of an already mildly dehydrated patient, leading to water retention, hypertension, and heart failure decompensation. They can also increase the risk for gastrointestinal bleeding, particularly in elderly patients with chronic hypoxia receiving anticoagulants. Finally, even if the newest cyclooxygenase- 2 selective NSAIDs are considered, the risk for acute cardiovascular events, which is high in elderly men with type 2 diabetes and ischemic heart disease, does not make them a safer option.

Low-dose colchicine is primarily recommended for longterm prophylaxis against new flares and may be advantageous over NSAIDs for this purpose $[18,19]$. It is also recommended for the treatment of recent onset ( $<36$ hours) acute arthritis [31], even without the need for high and usually intolerable doses recommended in the past [18]. Furthermore, in a clinical trial involving patients with stable coronary artery disease, the addition of colchicine $(0.5 \mathrm{mg} /$ day $)$ to standard therapy improved cardiovascular outcomes. Although these findings need to be replicated in patients with gouty arthritis, these results may offer another advantage of low-dose colchicine prophylaxis in patients with gouty arthritis [31].

A short course of oral glucocorticoids may also be useful for the resolution of an acute attack and can be co-administered safely with colchicine. However, the known long-term toxicity of glucocorticoids limits their use for prophylaxis [19,28]. In our patient, the initial colchicine/methylprednisolone combination for the treatment of acute attack was favored, because it was felt that low-dose colchicine alone could not achieve remission in a patient who was overweight and whose gout flare had been ongoing for several days.

ULT should begin at or soon after anti-inflammatory prophylaxis initiation. The target serum urate level should be $<6 \mathrm{mg} /$ $\mathrm{dL}$ or, in severe disease, $<5 \mathrm{mg} / \mathrm{dL}$ [24,28]. An xanthine oxidase inhibitor (ie, allopurinol, febuxostat) is the first-line drug class for ULT. The ACR recommendations do not favor either agent over the other [24]; however, the recent EULAR recommendations advocate the use of allopurinol as first line in patients with normal renal function [28]. When prescribing allopurinol, particularly to patients with renal impairment, caution should be taken in choosing the starting dose and subsequently in raising it, to avoid hypersensitivity; however, this has recently been debated [28,32]. As for the allopurinol versus febuxostat comparison, in a meta-analysis, febuxostat was more effective in achieving the $<6 \mathrm{mg} / \mathrm{dL}$ urate target level compared with allopurinol. However, this difference did not result in a similar benefit in the incidence of gout flares. Febuxostat was associated with a slightly, but significantly, lower incidence of adverse events compared with allopurinol [33].

Because our patient had a very high baseline serum urate level, febuxostat was chosen based on data from a phase III trial in which $36 \%$ of patients with baseline serum urate levels $\geq 10$ $\mathrm{mg} / \mathrm{dL}$ who received febuxostat achieved a serum urate level $<6 \mathrm{mg} / \mathrm{dL}$ in the final 3 monthly measurements, compared with only $10 \%$ of patients who received allopurinol [34]. Additionally, febuxostat was expected to act faster than allopurinol, because there is no need to start at low doses and titrate up, as is suggested with allopurinol. In our patient, almost 1 month of febuxostat $80 \mathrm{mg}$ daily lowered serum urate by $3 \mathrm{mg} / \mathrm{dL}$. However, this swift decline may have triggered the subsequent arthritis attacks despite the concomitant low-dose colchicine. This appears to have also occurred during the aforementioned trial in which withdrawals due to gout flares were more common in febuxostat- than in allopurinol-treated patients [34].

Independently of whether the new attacks are the result of an abrupt decline of serum urate or the still elevated urate levels, it is the anti-inflammatory treatment that clearly failed in the case described. Therefore, an alternative treatment option should be considered for patients with frequent gouty arthritis attacks who are not being successfully managed with standard-of-care medication.

The recognition that IL-1 $\beta$ plays a key role in the pathogenesis of MSU-induced inflammation led to the trials of IL-1 $\beta$ inhibition in patients with gouty arthritis, offering an alternative therapy in the difficult-to-treat patient. To date, 3 anti-IL-1 biologic agents have been evaluated in patients with gouty arthritis, either in small, open-label, retrospective studies, such as the IL-1 receptor antagonist anakinra $[7,35]$ or in randomized, controlled trials, such as the IL- 1 trap rilonacept $[5,36]$ and the anti-IL-1 $\beta$ monoclonal antibody canakinumab [6]. Today, only canakinumab is approved in the European Union for the treatment of adult patients with frequent gouty arthritis attacks (at least 3 in the previous 12 months) in whom NSAIDs and colchicine are contraindicated, are not tolerated, or do not provide an adequate response, and in whom repeated courses of corticosteroids are not appropriate.

Canakinumab has been studied in patients with gouty arthritis who have multiple comorbidities and failure, intolerance or contraindication to NSAIDs and/or colchicine in 2 phase III studies. In these 3-month clinical trials with 12 -week extensions, canakinumab $150 \mathrm{mg}$ proved more effective than triamcinolone $40 \mathrm{mg}$ for the treatment of acute gout and the prevention of new gout flares. However, adverse events were reported in $66 \%$ of canakinumab-treated patients compared with $53 \%$ in triamcinolone-treated patients over 24 weeks; most were mild or moderate. Adverse events reported more frequently with canakinumab included infections, low neutrophil count, and low platelet count [6].

The next important step in the management plan for the case presented herein is a discussion with the patient to assess the severity of symptoms and to balance the benefits versus risks of the addition of anti-cytokine therapy versus the on-demand use of short courses of glucocorticoids, until the serum urate target level of $<6 \mathrm{mg} / \mathrm{dL}$ is achieved. The target serum urate level should be aggressively pursued and may require frequent serum urate level measurements and optimization of ULT, by increasing the febuxostat dose and potentially adding a uricosuric agent. Whichever anti-inflammatory treatment is selected, only a sustained decrease of serum urate to optimal levels will keep the patient symptom-free in the long term and prevent tophus formation. Furthermore, in patients with difficult-to-treat gouty arthritis associated with major comorbidities, the importance of an interdisciplinary approach cannot be over emphasized. 


\section{An Innovative Approach to Managing a Patient With Complicated Gouty Arthritis and Chronic Kidney Disease}

\subsection{Introduction}

The incidence of chronic kidney disease (CKD) is rising rapidly worldwide, resulting in significant clinical, economic, and social costs [37]. Hyperuricemia is increasingly recognized as a risk factor for renal failure progression. Moreover, increased serum uric acid levels may be linked to cardiovascular disease and mortality in patients with CKD [38].

Gouty arthritis, a painful and potentially disabling form of arthritis, is caused by the overproduction of urate or, more commonly, by renal urate underexcretion. The condition leads to monosodium urate crystals deposition in the joints caused by longstanding hyperuricemia. Gouty arthritis is generally characterized by intense episodes of painful swelling in single joints, most often in the big toe, that appear red and warm [39]. Tissue deposition of urate crystals in the interstitial space of the kidneys can also lead to renal failure as a result of supersaturation of extracellular fluids with urate [40].

\subsection{Case Report}

A 74-year-old man was admitted to our hospital with persistent diarrhea, vomiting, and reduction of urine output (despite taking furosemide $75 \mathrm{mg}$ /day). He also reported arthralgias with recurrent attacks of pain, particularly in his hand joints, and a persistent left lumbar colic pain.

Thirty years ago, the patient was diagnosed with type 2 diabetes and hypertension, which was currently being treated with an angiotensin-converting enzyme inhibitor (ACE-I) and $\beta$-blockers (ramipril $5 \mathrm{mg} /$ day; metoprolol $50 \mathrm{mg} /$ day). He had a cardiologic history of aortic valvular stenosis and coronary artery disease with unstable angina. Two months before presentation, he was diagnosed with non-Q-wave myocardial infarction, without undergoing coronary angiography due to several comorbidities. The patient also has stage $3 \mathrm{CKD}$, and 4 years before had an episode of acute kidney injury (AKI) with anuria that required 2 hemodialysis treatments. He also had a history of gouty arthritis and had been self-medicating with nonsteroidal anti-inflammatory drugs (NSAIDs) for 4 years to manage his symptoms.

On admission, the patient's blood pressure was $110 / 70 \mathrm{~mm} \mathrm{Hg}$, he appeared dehydrated, and reported losing almost 9 pounds ( 4 $\mathrm{kg}$ ) during the past week. Upon physical examination, tophaceous gout lesions at his metacarpophalangeal joints were noted.

Results of the patient's laboratory tests were as follows: serum creatinine $=1.68 \mathrm{mg} / \mathrm{dL}$, with an estimated glomerular filtration rate of $39 \mathrm{~mL} / \mathrm{min}$ (calculated by the Chronic Kidney Disease Epidemiology Collaboration formula); albuminuria = $680 \mathrm{mg} /$ day; serum potassium $=3.2 \mathrm{mEq} / \mathrm{L}$; serum bicarbonate $\left(\mathrm{HCO}_{3}\right)=18 \mathrm{mmol} / \mathrm{L}$; serum uric acid $=10.8 \mathrm{mg} / \mathrm{dL}$; C-reactive protein $(\mathrm{CRP})=8.5 \mathrm{mg} / \mathrm{dL}$; blood glucose $=174 \mathrm{mg} / \mathrm{dL}$; and glycosylated hemoglobin $=8.4 \%$. Renal ultrasound examination showed bilateral renal stones (on the left more than the right side) with left renal hydronephrosis; renal cortical thickness was within normal limits.

Current treatment options to control pain and inflammation of acute and chronic gouty arthritis include NSAIDs, colchicine, and corticosteroids (Table 2). However, NSAIDs should not be used in patients with acute kidney injury [40], and colchicine therapy is associated with significant side effects, namely gastrointestinal (GI) side effects. Despite presenting with GI symptoms on admission, our patient was initially treated with low-dose allopurinol and colchicine. After 2 days, allopurinol was stopped due to hypersensitivity characterized by leukocytoclastic vasculitis with cutaneous raised palpable petechiae. Of note, diuretics and amoxicillin (added to our patient's regimen due to a concomitant urinary tract infection) are believed to increase the risk of hypersensitivity to allopurinol. Additionally, colchicine, despite given at a low dose, was stopped after 2 days due to the patient experiencing nausea and persistent diarrhea. The patient was subsequently placed on oral prednisone $25 \mathrm{mg} /$ day with tapering; his serum glucose levels were tightly controlled after the optimization of insulin therapy.

Intravenous (iv) alcalization therapy with bicarbonate was added to raise the urinary $\mathrm{pH}$ and reduce the size of the uric acid renal calculi. Once the serum acid-base balance was re-established and the fluid challenge restored, serum creatinine levels improved. The patient was taken off the ACE-I and placed instead on the angiotensin receptor blocker losartan, which has been shown to produce a uricosuric effect. Treatment with uricase (rasburicase), an enzyme that oxidizes uric acid to a more soluble form and indicated for the treatment of hyperuricemia associated with tumor lysis syndrome, was introduced. However, after the second iv administration, the patient had a cutaneous allergic reaction. The treatment was therefore discontinued. It was hypothesized that rasburicase, which is a nonhuman protein, induced an allergic reactions, which had also occurred when the patient was previously given allopurinol.

After 5 days in the hospital, the patient was discharged home. His left lumbar pain was significantly reduced, and after the expulsion of the urinary microcalculi, ultrasound showed that the hydronephrosis was significantly improved. The pain in his hand joints was mitigated and his serum creatinine was stable at $1.58 \mathrm{mg} / \mathrm{dL}$. His serum uric acid level was slightly reduced but was still greater than $8 \mathrm{mg} / \mathrm{dL}$.

\subsection{Discussion}

Evidence suggests that end-stage renal disorder (ESRD) is characterized by a state of chronic inflammation linked to oxidative stress, endothelial dysfunction, vascular calcification, and wasting. Reports suggest that CKD is a chronic inflammatory disease, as it is now recognized that rapid kidney function loss in CKD is independently associated with increased baseline levels of

Table 2

Treatment goals in gouty arthritis

\begin{tabular}{ll}
\hline 1 & Terminate attacks \\
2 & Provide control of pain and inflammation \\
3 & Prevent future attacks \\
4 & $\begin{array}{l}\text { Prevent complications such as tophi, destructive } \\
\text { arthropathy, renal stones, and renal insufficiency }\end{array}$
\end{tabular}


CRP and soluble tumor necrosis factor receptor-2 [41]. Therefore, the attenuation of the inflammatory response to renal injury on vascular structures induced by statin therapy may explain the renoprotective actions of these agents in patients with CKD [42], and suggests that inflammation represents an important target for pathogenetic interventions both in AKI and the progression of CKD.

Recent evidence suggests that interleukin-1 (IL-1), a proinflammatory cytokine, plays a major role in mediating gouty arthritis inflammation [6,24,43-46]. Additionally, both experimental and human studies show that the NLRP3 inflammasome plays a role in the development of acute and chronic tubulo-interstitial disease [47]. Moreover, recent data show that inflammasome activation is related to proinflammatory effects of NLRP3 in autologous nephrotoxic serum nephritis, including NLRP3-mediated glomerular release of pro-inflammatory cytokines [48]. Therefore, therapeutic blockade of the NLRP3/ASC/IL-1 $\beta$ axis may also be beneficial in glomerulonephritis [49].

\section{What is the rationale for anti-IL-1 $\beta$ therapy in gouty arthritis and other chronic diseases?}

Acute gouty arthritis appears to be mediated by IL-1 $\beta$ that exerts strong proinflammatory activities. In a randomized, controlled trial, canakinumab - a fully humanized monoclonal anti-human IL-1 $\beta$ antibody - was more effective in reducing pain and risk of a new flare in patients with gouty arthritis as compared with triamcinolone acetonide [22].

Anti-IL-1 $\beta$ therapy is an alternative for patients with acute gouty arthritis, especially when NSAID and colchicine therapy is contraindicated, as was the case in our patient. Of note, canakinumab has no significant effect on renal impairment and no dose adjustment is necessary for patients with renal impairment [8]. Furthermore, the reduction of inflammation and gouty arthritis with anti-IL-1 $\beta$ therapy may provide an opportunity to increase urate-lowering therapy to an optimal dose - another reason as to why treatment with an IL-1 $\beta$ inhibitor may benefit our patient.

IL- $1 \beta$ expression has also been observed in $\beta$-cells of patients with type 2 diabetes, suggesting a potential role of IL- 1 in the pathogenesis of type 2 diabetes via increased insulin production, improved insulin sensitivity, and slowing of disease progression by increasing $\beta$-cell burden. The production and secretion of IL- $1 \beta$ from $\beta$-cells is reported to be induced by high glucose levels, inhibiting the function and promoting the apoptosis of $\beta$-cells [50]. These data may provide further insight into the cause of ESRD in patients with type 2 diabetes and provide a fascinating prospective therapy for patients with diabetes mellitus, the most common cause of ESRD and reason for requiring dialysis treatment in the United States.

IL-1 $\beta$ inhibition proved successful in one of our patients who had been diagnosed with type 2 diabetes and Muckle-Wells syndrome (MWS), a rare hereditary autoinflammatory disorder, with accompanying renal amyloidosis AA and renal impairment. We initiated sc anakinra $100 \mathrm{mg} /$ day. After only 5 days of treatment, the patient had significant improvement in glycemic control and levels of the inflammatory marker serum amyloid A (SAA), which decreased from $4.5 \mathrm{mg} / \mathrm{dL}$ to $0.5 \mathrm{mg} / \mathrm{dL}$. Additionally, her serum creatinine level decreased from $4.5 \mathrm{mg} / \mathrm{dL}$ to $3.8 \mathrm{mg} / \mathrm{dL}$ and the estimated glomerular filtation rate (GFR) increased from $10 \mathrm{~mL} /$ min to $13 \mathrm{~mL} / \mathrm{min}$ (personal unpublished data).

We recently published a report that corroborated the benefits of anti-IL-1 $\beta$ therapy in renal disease associated with MWS. Two patients, father and son, aged 52 and 26 years, respectively, with genetically-confirmed MWS with amyloid A (AA) deposits on renal biopsy, received canakinumab every 60 days over a period of 18 months. Treatment resolved inflammatory symptoms, normalized SAA levels, and markedly improved GFR and nephrotic proteinuria, with no adverse events. These data, although limited to 2 patients, emphasize that therapeutic intervention with canakinumab suppresses both inflammation and IL-1 $\beta$-mediated manifestations, and can contribute to improved kidney function in patients with MWS who have overt renal amyloidosis, with no adverse event after 36 months of treatment [51].

Despite the promising clinical results achieved with antiIL-1 $\beta$ therapy in patients with difficult-to-treat gouty arthritis, some would argue that the costs associated with this therapy is prohibitive. However, it is important to take into consideration the overall costs involved in managing acute gout attacks and the costs for repeated hospitalizations when patients are managed unsuccessfully.

\section{Contributors}

Wolfgang A. Schmidt and Annalina Avram are the authors of clinical case: "IL-1 Inhibition Inflammatory Syndrome in Systemic Due to Gouty Arthritis".

Marie José Santos is the author of clinical case: "Anti-IL-1 Therapy Suppresses Inflammation and Provides an Opportunity to Optimize ULT in a Patient with Difficult-to-Treat Gouty Arthritis".

Catia Duarte is the author of clinical case: "Treatment Dilemma in Gouty Arthritis: Managing a Patient With Complex Comorbidities and Intolerance to Standard Therapies."

Charalampos Papagoras, Panagiotis Skendros, Konstantinos Ritis are the authors of clinical case: "Managing a Patient With Persistent Gout Flares Complicated by Comorbidities and Polypharmacy: An Evidence-based Approach."

Roberto Scarpioni is the author of clinical cases: "An Innovative Approach to Managing a Patient With Complicated Gouty Arthritis and Chronic Kidney Disease" and "What is the rationale for anti-IL-1 $\beta$ therapy in gouty arthritis and other chronic diseases?"

\section{Disclosures of interest}

A. Avram: The author has received honoraria from Novartis

C. Duarte: The author has nothing to disclose.

M. José Santos: The author has received research grants from Abbvie, MSD, and Pfizer, and speaker's fees and/or consulting fees from Abbvie, BMS, Janssen, MSD, Pfizer, and Roche.

C. Papagoras: The author has nothing to disclose.

K. Ritis: The author has nothing to disclose.

R. Scarpioni: The author has received a speaking fee from Novartis and grant for training or teaching events from Sanofi. 
W.A. Schmidt: The author has received honoraria from Berlin-Chemie and Novartis.

P. Skendros: The author has received an honorarium from Novartis for participation in an advisory board.

\section{References}

[1] Nicholls DW, Rajapakse CN. Systemic inflammatory response syndrome (SIRS) from acute polyarticular gout. N Z Med J 1999;112:434-5.

[2] Huppertz A, Hermann KG, Diekhoff T, et al. Systemic staging for urate crystal deposits with dual-energy CT and ultrasound in patients with suspected gout. Rheumatol Int 2014;34:763-71.

[3] Gutierrez M, Schmidt WA, Thiele RG, et al. International consensus for ultrasound lesions in gout: results of Delphi process and webreliability exercise. Rheumatology (Oxford) 2015;54:1797-805.

[4] Latourte A, Bardin T, Richette P. Prophylaxis for acute gout flares after initiation of urate-lowering therapy. Rheumatology (Oxford) 2014;53:1920-6.

[5] Sundy JS, Schumacher HR, Kivitz A, et al. Rilonacept for gout flare prevention in patients receiving uric acid-lowering therapy: results of RESURGE, a phase III, international safety study. J Rheumatol 2014;41:1703-11.

[6] Schlesinger N, Alten RE, Bardin T, et al. Canakinumab for acute gouty arthritis in patients with limited treatment options: results from two randomised, multicentre, active-controlled, double-blind trials and their initial extensions. Ann Rheum Dis 2012;71:1839-48.

[7] Ottaviani S, Moltó A, Ea HK, et al. Efficacy of anakinra in gouty arthritis: a retrospective study of 40 cases. Arthritis Res Ther 2013;15:R123.

[8] European Medicines Agency (EMA), Committee for Medicinal Products for Human Use, January 17, 2013 available at http://www.ema.europa.eu/docs/en_GB/document_library/ Summary_of_opinion/human/001109/WC500137458.pdf. Accessed January 23, 2015.

[9] Kuo CF, Grainge MJ, Mallen C, et al. Rising burden of gout in the UK but continuing suboptimal management: a nationwide population study. Ann Rheum Dis 2014 Jan 15. doi:10.1136/ annrheumdis-2013-204463. [Epub ahead of print]

[10] Smith E, Hoy D, Cross M, et al. The global burden of gout: estimates from the Global Burden of Disease 2010 study. Ann Rheum Dis 2014;73:1470-6.

[11] Kuo CF, Grainge MJ, Mallen C, et al. Comorbidities in patients with gout prior to and following diagnosis: case-control study. Ann Rheum Dis 2014 Nov 14. doi:10.1136/annrheumdis-2014-206410. [Epub ahead of print]

[12] Juraschek SP, Kovell LC, Miller ER 3rd, et al. Gout, urate lowering therapy and uric acid levels among US adults. Arthritis Care Res (Hoboken) 2014 Sep 8. doi:10.1002/acr.22469. [Epub ahead of print]

[13] Harirforoosh S, Asghar W, Jamali F. Adverse effects of nonsteroidal antiinflammatory drugs: an update of gastrointestinal, cardiovascular and renal complications. J Pharm Pharm Sci 2013;16:821-47.

[14] Araújo F, Cordeiro I, Teixeira F, et al. Portuguese recommendations for the diagnosis and management of gout. Acta Reumatol Port 2014;39:158-71.

[15] So A, De Smedt T, Revaz S, et al. A pilot study of IL-1 inhibition by anakinra in acute gout. Arthritis Res Ther 2007;9:R28.

[16] Shmerling RH. Management of gout: a 57-year-old man with a history of podagra, hyperuricemia, and mild renal insufficiency. JAMA 2012;28;308:2133-41.

[17] Sivera F, Andrés M, Carmona L, et al. Multinational evidencebased recommendations for the diagnosis and management of gout: integrating systematic literature review and expert opinion of a broad panel of rheumatologists in the $3^{\mathrm{e}}$ initiative. Ann Rheum Dis 2014;73:328-35.

[18] Zhang W, Doherty M, Bardin T, et al. EULAR evidence based recommendations for gout. Part II: Management. Report of a task force of the EULAR Standing Committee for International Clinical Studies Including Therapeutics (ESCISIT). Ann Rheum Dis 2006;65:1312-24.

[19] Khanna D, Khanna PP, Fitzgerald JD, et al. 2012 American College of Rheumatology guidelines for management of gout. Part 2: therapy and antiinflammatory prophylaxis of acute gouty arthritis. Arthritis Care Res (Hoboken) 2012;64:1447-61.

[20] Ahern MJ, Reid C, Gordon TP, et al. Does colchicine work? The results of the first controlled study in acute gout. Aust N Z J Med 1987; 17:301-4.

[21] Terkeltaub RA, Furst DE, Bennett K, et al. High versus low dosing of oral colchicine for early acute gout flare: Twenty-four-hour outcome of the first multicenter, randomized, double-blind, placebo-controlled, parallel-group, dose-comparison colchicine study. Arthritis Rheum 2010;62:1060-8.

[22] Schlesinger N, De Meulemeester M, Pikhlak A, et al. Canakinumab relieves symptoms of acute flares and improves health-related quality of life in patients with difficult-to-treat Gouty Arthritis by suppressing inflammation: results of a randomized, dose-ranging study. Arthritis Res Ther 2011;13:R53.

[23] So A, De Meulemeester M, Pikhlak A, et al. Canakinumab for the treatment of acute flares in difficult-to-treat gouty arthritis: Results of a multicenter, phase II, dose-ranging study. Arthritis Rheum 2010;62:3064-76.

[24] Khanna D, FitzGerald JD, Khanna PP, et al. 2012 American College of Rheumatology Guidelines for Management of Gout Part I: Systematic Non-pharmacologic and Pharmacologic Therapeutic Approaches to Hyperuricemia. Arthritis Care Res (Hoboken) 2012;64:1431-46.

[25] Becker MA, Baraf HSB, Yood RA, et al. Long-term safety of pegloticase in chronic gout refractory to conventional treatment. Ann Rheum Dis 2013;72:1469-74.

[26] Richette P, Bardin T. Gout. Lancet 2010;375:318-28.

[27] Mitroulis I, Kambas K, Chrysanthopoulou A, et al. Neutrophil extracellular trap formation is associated with IL-1 $\beta$ and autophagy-related signaling in gout. PLoS One 2011;6:e29318.

[28] Richette P, Doherty M, Pascual E, et al. Updated EULAR evidence-based recommendations for the management of gout. Ann Rheum Dis 2014;73(suppl 2):783.

[29] Roddy E, Mallen CD, Hider SL, et al. Prescription and comorbidity screening following consultation for acute gout in primary care. Rheumatology (Oxford) 2010;49:105-11.

[30] De Vera MA, Marcotte G, Rai S, et al. Medication adherence in gout: a systematic review. Arthritis Care Res (Hoboken) 2014;66:1551-9.

[31] Nidorf SM, Eikelboom JW, Budgeon CA, et al. J Am Coll Cardiol 2013;61:404-10.

[32] Ramasamy SN, Korb-Wells CS, Kannangara DR, et al. Allopurinol hypersensitivity: a systematic review of all published cases, 1950-2012. Drug Saf 2013;36:953-80.

[33] Faruque LI, Ehteshami-Afshar A, Wiebe N, et al. A systematic review and meta-analysis on the safety and efficacy of febuxostat versus allopurinol in chronic gout. Semin Arthritis Rheum 2013;43:367-75.

[34] Schumacher HR Jr, Becker MA, Wortmann RL, et al. Effects of febuxostat versus allopurinol and placebo in reducing serum urate in subjects with hyperuricemia and gout: a 28-week, phase III, randomized, double-blind, parallel-group trial. Arthritis Rheum 2008;59:1540-8.

[35] Ghosh P, Cho M, Rawat G, et al. Treatment of acute gouty arthritis in complex hospitalized patients with anakinra. Arthritis Care Res (Hoboken) 2013;65:1381-4.

[36] Schumacher HR Jr, Sundy JS, Terkeltaub R, et al. Rilonacept (Interleukin-1 Trap) in the prevention of acute gout flares 
during initiation of urate-lowering therapy: results of a phase II randomized, double-blind, placebo-controlled trial. Arthritis Rheum 2012;64:876-84.

[37] Scarpioni R, Ricardi M, Melfa L, et al. Dyslipidemia in chronic kidney disease: are statins still indicated in reduction cardiovascular risk in patients on dialysis treatment? Cardiovasc Ther 2010;28:361-8.

[38] Jalal DI, Chonchol M, Chen W, et al. Uric acid as a target of therapy in CKD. Am J Kidney Dis 2013;61:134-46.

[39] Neogi T. Gout. N Engl J Med 2011;364:443-52.

[40] Praga M, Sevillano A, Auñón P, et al. Changes in the aetiology, clinical presentation and management of acute interstitial nephritis, an increasingly common cause of acute kidney injury. Nephrol Dial Transplant 2014 Oct 16. pii: gfu326. [Epub ahead of print]

[41] Nakanishi I, Moutabarrik A, Okada N, et al. Interleukin-8 in chronic renal failure and dialysis patients. Nephrol Dial Transplant 1994;9:1435-42.

[42] Scarpioni R, Ricardi M, Albertazzi V, et al. Treatment of dyslipidemia in chronic kidney disease: Effectiveness and safety of statins. World J Nephrol 2012;1:184-94.

[43] Schlesinger N. Anti-interleukin-1 therapy in the management of gout. Curr Rheumatol Rep 2014;16:398.

[44] Perez-Ruiz F, Chinchilla SP, Herrero- Beites AM. Canakinumab for gout: a specific, patient-profiled indication. Expert Rev Clin Immunol 2014;10:339-47.
[45] Khanna P, Gladue HS, Singh MK, et al. Treatment of acute gout: A systematic review. Semin Arthritis Rheum 2014;44:31-8.

[46] Martinon F, Pétrilli V, Mayor A, et al. Gout-associated uric acid crystals activate the NALP3 inflammasome. Nature 2006;440:237-41.

[47] Fang L, Xie D, Wu X, et al. Involvement of Endoplasmic Reticulum Stress in Albuminuria Induced Inflammasome Activation in Renal Proximal Tubular Cells. PLoS ONE 2013;8:e72344.

[48] Scarpioni R, Ricardi M, Albertazzi V. Secondary amyloidosis in autoinflammatory diseases, the role of inflammation in renal damage. World J Nephrol 2015. In press.

[49] Andersen K, Eltrich N, Lichtnekert J, et al. The NLRP3/ASC inflammasome promotes T-cell-dependent immune complex glomerulonephritis by canonical and noncanonical mechanisms. Kidney Int 2014;86:965-78.

[50] Larsen CM, Faulenbach M, Vaag A, et al. Interleukin-1receptor antagonist in type 2 diabetes mellitus. $\mathrm{N}$ Engl J Med 2007;356:1517-26

[51] Scarpioni R, Rigante D, Cantarini L, et al. Renal involvement in secondary amyloidosis of Muckle-Wells syndrome: marked improvement of renal function and reduction of proteinuria after therapy with human anti-interleukin-1 $\beta$ monoclonal antibody canakinumab. Clin Rheumatol Clin Rheumatol 2015;34:1311-6. 